\title{
وسيلة الأوراق الممزقة وتأثيرها على مهارة القراءة: دراسـة تجريبية في معهد تو انكو لنتاو العصري المتحدي
}

\author{
Awalul Fajri \\ e-mail: awalulfajri.nagsos@gmail.com \\ Madrasah Ibtidaiyah Negeri 3 Kota Padang
}

ملخص: الغرض لهذا البحث هو لمعرفة تأثير استعمال وسيلة الأوراق الممزقة على مهارة قراءة النصوص العربية في معهد توانكو لنتاو العصري المتحدي بمحاظة تانة داتر.أما منهج البحث المستعمل فهو البحث شبه التجريبي مع عينة البحث ع تلميذا المقسمين إلى الفرقتين، ـ ت تلميذا للفرقة التجريبية و الباقياة منهم للفرقة الضبطية. استعمل هذا البحث طريقة اختبارية مع تحليل إحصائى فى تحليل البيانات. دلت نتيجة البحث أن استعمال وسيلة الأوراق الممزقة لله آثار على مهارة قراءة النصوص العربية ودلّ عليها تفريق نتيجة التلاميذ فى قراءة النصوص العربية بين الفصل التجريبى و الفصل الضبطي.

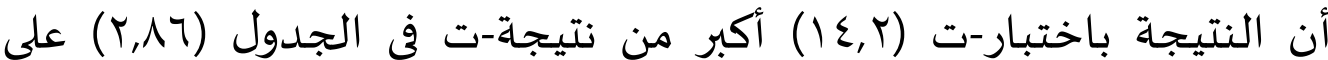

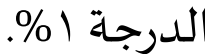

\section{الكلمات الأسـاسية: الأوراق الممزقة، مهارة القراءة، المعهد العصري، الوسيلة.}

\begin{abstract}
The purpose of this study was to determine the effect of the use of snippets text media on Arabic reading proficiency at Integrated Modern Islamic Boarding School Tuanku Lintau, Tanah Datar regency. The research used a quasi-experimental method. The population was 40 students who were divided into an experimental group of 20 students and a control group of 20 students. Data collection technique was a test. Then, the data were analyzed using t-test. The result of this research described that the use of snippets text media has an effect on Arabic reading proficiency. This was indicated by the difference of students' learning outcomes between the experimental and control group. It was found that t-calculated 14.2 was higher than t-table 2, 86 at $1 \%$ significance level.
\end{abstract}

Key Words: Snippets text, reading proficiency, modern Islamic boarding school, media.

إن التعليم هو شئ ضروري في حياة

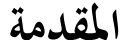

الفرد والمجتمع، لأن التعليم يرشد

الناس من الجهل إلى المعرفة والعلوم 
المادة المكتوبة (صينى و عبد الله، 1919 :

لذلك اتضح أن المهارة اللغوية هي

أبسط النشاط اللغوى الذى يؤدى إلى الى

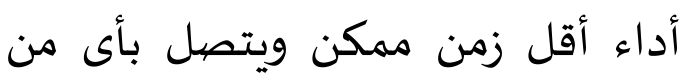

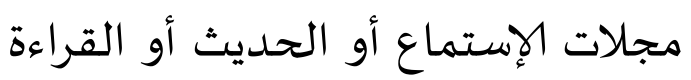

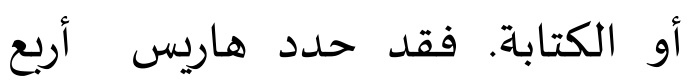

مكونات لكل مهارة من المهارات الأربع :

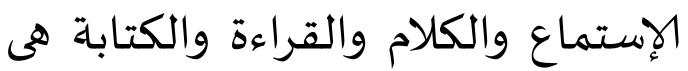

الأصوات والحستهاع والحروف والتركيبات

والمفردات ومعدل السرعة (يونس و عبد

الرؤوف ، r. . . OV-00: ).

كما هو المعروف أن مهارة القراءة

هي مهارة اللغوية الثالثة بعد مهارة

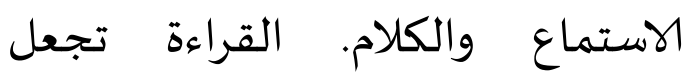

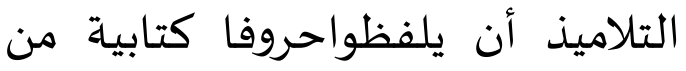
اللغة المدروسة ويفهموا علامة الترقيم، مئحس مثل :الشولة، والنقطة وغيرها. كما

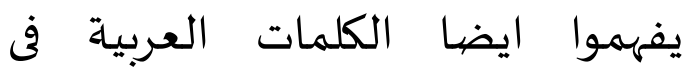
النصوص وتراكيها ومعانها المقروءة. أن أن إنهوبهات أهم مهارة الغوية في المستوى اللغوي

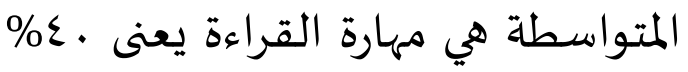

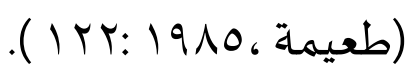

أما لفظ وسيلة أو ميديا (Media)

فمأخوذة من اللغة اللاتينية الميديوس اوسياسيا وهو لغة الوسط أوالوسيلة،
الكثيرة في هذه الحياة، ولا يستطيع الناس أن يعيش بدون التعليم، كلما

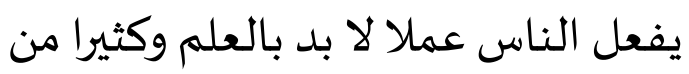
العلوم نجدها من التعليم إما العلوم

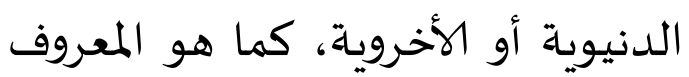
أن أحد أغراض التعليم هو إيجادة الاحرونها هو المعروف

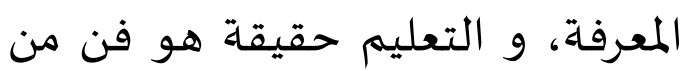
فنون إيصال المادة الدراسية من المعلم إلى أذهان التلاميذ وتغيير أعمالهم، من إنهن

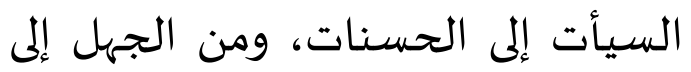

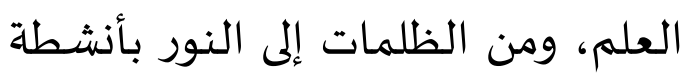
تعليمية معينة وبطريقة مناسبة وبأقل

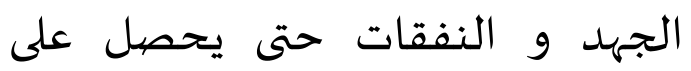
أغراض التعليم الكافية والكاملة. فالمعلم الناجح في حقيقتا هو طريقة ناجحة توصل المادة الدراسية إلى أذهان التلاميذ في عملية التعليم. وتعليم القراءة يعنى تطوير لئمليه مجموعة من العادات الجسدية

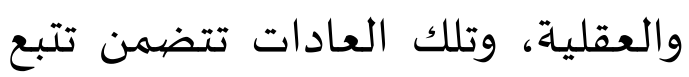

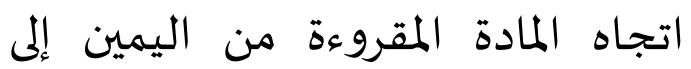
اليسار كما هو الحال في اللغة العربية وتمييز الحروف والمقاطع والكلمات والتعبيرات والجمل مع إدراك معانيها، وذلك حتى يتم الأفكار التى تشتمل عليها 
وهذه الآراء مناسبة أيضا بقول حامالك (dalam Arsyad, 2005: 15) الوسائل في التعليم يبعث الرغبة في إثارة الاهتمام الجديدة، والدافع لأنشطة

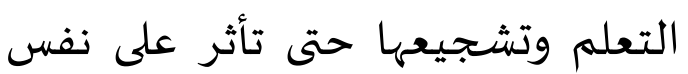

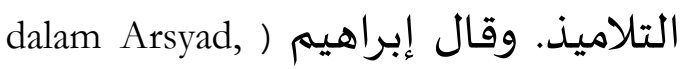

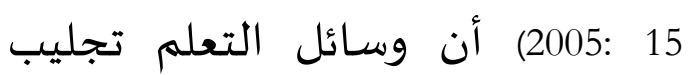
السرور للتلاميذ وتجدّد نشاطهم وأنها تساعد علي تثبيت الحقائق في أذهان التلاميذ وأنها تحيي الدرس. تسئل

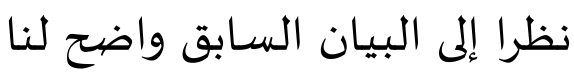
أن المدرس لابد له أن يستخدم الوسائل التعليمية المناسبة في تعليم لاسيام في

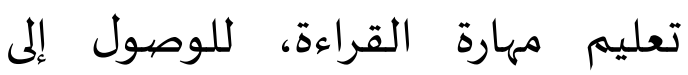
أغراض التعليم الكافية والكاملة وترقية

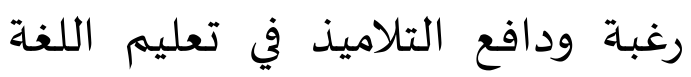
العربية. وفي هذا الزمن ، لقد ظهر لنا

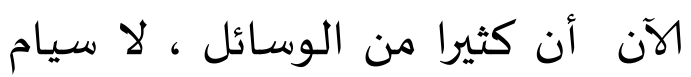
وسائل تعليم التي يتعلق بمهارة القراءة،

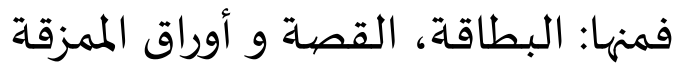

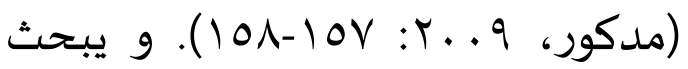

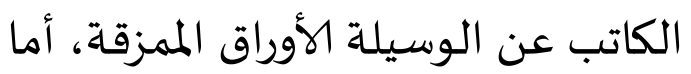

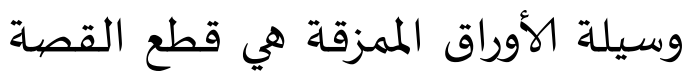
التي يقدم توصية ليسهل الطلاب في

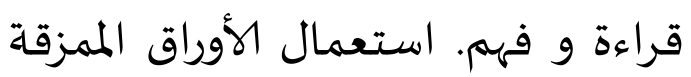
ليسهل الطلاب تقديم الفكرة باللغة
ويذكر Media كثيرا في اللغة العربية بلفظ الوسائل جمعا من الوسيلة لتنقيل شيء عن الأشياء لغة كانت أم أمناء

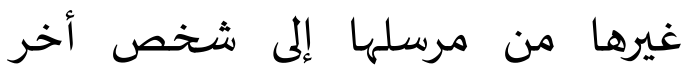

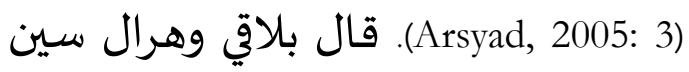
أن الوسائل هي الآلات الوسائلية المستعملة لآداء أو لإيصال شيئ

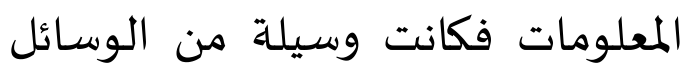
أوالطريقة أوالآت من المعلومات الماشية بين المكالمين أوالمكلمين وتلك المعلومات. لتحقيق الأهداف التعلمياة يحتاج المعلم إلى الوسيلة التعليمية كما قال

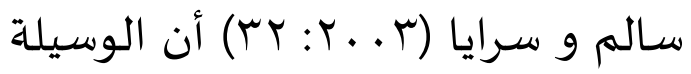

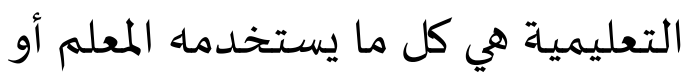

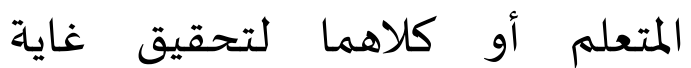

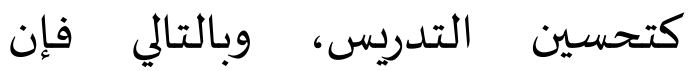
الوسائل ليست غايات في حد ذاتها، بل هي أدوات لتحقيق تلك الغايات، والوسائل التعليمية هي المواد والأجهزة والمواقف التي تحمل الرسالة التعليمية

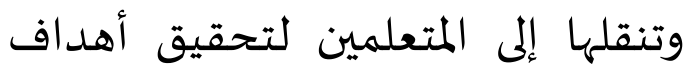
تعليمية محددة. ولقد تعددت السميات التى لتحده أطلقت على مفهوم الوسائل التعليمية

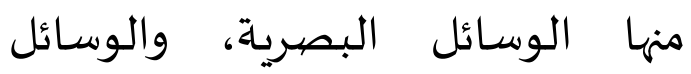
السمعياة، والوسائل السمعية البصرية. 


\begin{tabular}{|c|c|c|}
\hline \multicolumn{3}{|c|}{ الجدول الأول } \\
\hline$V \cdot-$ & $v \cdot+$ & ججتمع \\
\hline$r$. & 0 & To \\
\hline
\end{tabular}

نظرا على البينات السابقة واضح لنا أن نتيجة مهارة القراءة في هذ المعهد بعيدة جدا عن المطلوب. وهذا يستدلّ بوجود حصول الاختبار اليومي للتلاميذ

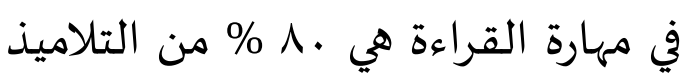

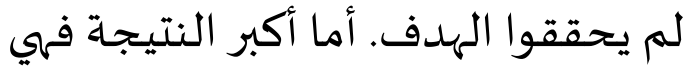
1أصغر النتيجة فهي مب، والنتيجة المعيارية لتعليم اللغة العربية هي . V. أما التلاميذ الذين لم يصلوا إلى النتيجة لماليه المعيارية فهو . ^ \% والذين يصلون إلى الى النتيجة المعيارية فهو .r \% \% بذلك، نتيجة التعليم للتلايذ الذين لم يصلوا إلى النتيجة المعيارية أكثر من نتيجة التعليم للتلايذ الذين يصلون إلى النتيجة المعيارية. فلذلك، البيانات السابقة دليل واضح لنا أن التلاميذ لم المئه يستطيعوا قراءة النصوص العربية

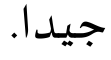

\section{طريقة البحث}

هذا البحث هو البحث الميداني

الكمي في النوع التجريبي، والطريقة المستعملة هي طريقة إحصائية لأن البحث يدور حول تعليم مهارة القراءة
Acep ) الأجنبية وقراءة نصيوصيه

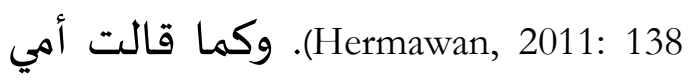
محمودة أن وسيلة الأوراق الممزقة طلب وحمات المات التلاميذ بها أن يفكر صحيح لترديب

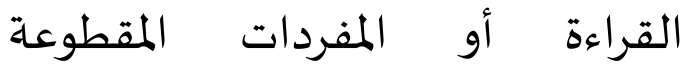
،(Machmudah \& Rosyidi, 2008: 159-160) كما ذهب إليه أزهار أرشاد ( Arsyad,

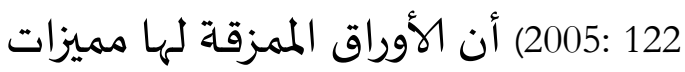
كثيرة في تعليم مهارة القراءة، وهذه الوسيلة تستطيع لتساعد التلاميذ في تدريب مهارة القراءة. وبعدما قابل الكاتب مع مدرّسة فهاءة اللغة العربية في المعهد، فرأى الباحث

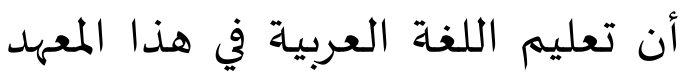
غير ممتعاة. ومواد التعليمية التي تعلم التلاميذ منها: التعرف، البيت، المقصف،

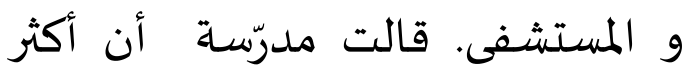
التلاميذ لم يستطيعوا قراءة النصوص المسئه العربية جيدا. أما بنسبة إلى نتيجة المحصيولة من تعليم مهارة القراءة في هذا المعهد بعيدة عن المطلوب، أما هذه النتيجة تتحلى في الاختبارات اليومية في تعليم مهارة القراءة، أما جهاة نتيجة من تعليم

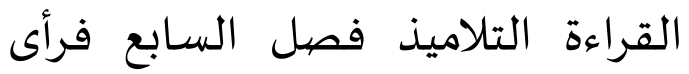

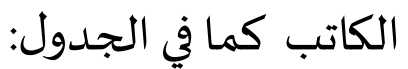




$$
\text { الإيضاح: }
$$

معدل من فرق الدرجة : : M

الأخطاء المعياري عنلى : SE

$$
\text { معدل من فرق الدرجة }
$$

\section{نتيـجة البحث ومناقشتها}

غرض البحث لتعرف ت تأثير

وسيلة الأوراق الممزقة على مهارة القراءة في معهد توانكو لنتاو العصري المتحدى. يريد الباحث أن ينظر نتيجة تعليم مهارة القراءة (في معهد توانكو لنتاو

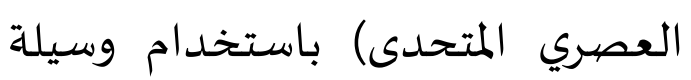
الأوراق الممزقة وتفرقه بنتيجة تعليم مهارة القراءة بدون استخدام وسيلة الأوراق الممزقة. ومن هنا يوضح الباحث لهراءه بدون استحام وسيله

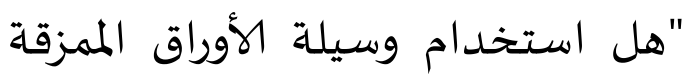
يستطيع أن يؤثر على مهارة القراءة في معهد توانكو لنتاو العصري المتحدى أم على ميارة المراعة في

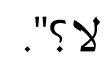

إضيافة إلى ذلك، قد قام الباحث

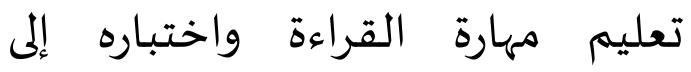
الفصلين في معهد توانكو لنتاو العصري

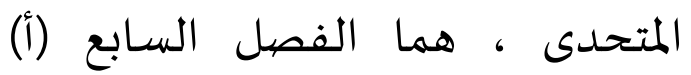
كالفصل التجريبي (experiment group) و الفصل السابع (ب) كالفصل الضبطي .control group)
باستخدم وسيلة الأوراق الممزقة في المعهد توانكو لنتاو العصري المتحدى باسيل (دراسة تجريبية). أما عينة البحث فهي التلاميذ في الفصل السابع (أ) والفصل السابع (ب) الذين لهم قدرة متساوية في مهارة

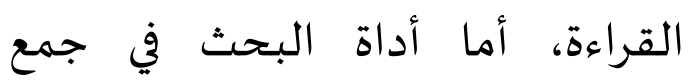
البيانات فهو الاختبار الذي يجري اهني بتقديم الأسئلة لمعرفة نتيجة مهارة المتبار الميات قراءة التلاميذ باستخدام وسيلة الأوراق

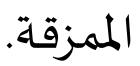
إن تصميم هذا له البحث باستعمال تصميم شباء التجريبي، والذي يقوم على تجريبة جديدة في الفرقة الأولى والفرقة الثانية بغير تجربه استخدام وسيلة الأوراق الممزقة. أما تصميم البحث كما في الجدول التالي.

\begin{tabular}{|c|c|c|c|}
\hline$R$ & 01 & $X$ & 02 \\
\hline$R$ & 03 & - & 04 \\
\hline
\end{tabular}

الفرقة/ الفصل : R

الاختبار القبلي : (Pre-test)

التجريب:

الاختبار البعدي: 02 (Post-test) وفي إنهاء البيانات يستعمل الباحث الرموز التالي: - مئاء $\mathrm{t}_{\mathrm{o}}=\underline{\mathrm{M}}_{\mathrm{D}}$ $\mathrm{SE}_{\mathrm{MD}}$ 


$$
\mathrm{SE}_{\mathrm{MD}}
$$

وأما خطوات إحصيائيتها فهي ما

بحث الباحث عن فرق الدرجة

بين النتيجتين باستعمال الرمز:

$$
\mathrm{D}=\mathrm{X}-\mathrm{Y}
$$

ب. بحث الباحث عن فرق الدرجة (D)

$$
\text { حتى يحصل على }
$$

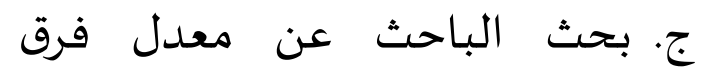

$$
\text { الدرجة باستعمال الرمز : بحت البـ }
$$

$$
\mathrm{M}_{\mathrm{D}}=\sum \frac{\mathrm{D}}{\mathrm{N}}
$$

D. ضياعف الباحث نتيجة التفريق D

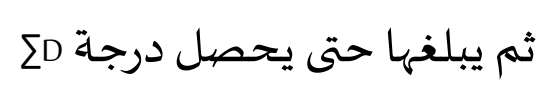

هـ بحث الباحث عن الإنحراف المعياري

Defiasi Standar dari ( عن فرق الدرجة

(Difference [SD $\left.{ }_{\mathrm{D}}\right]$

$$
S D_{D=\sqrt{ }} \sum_{N}-\frac{\left(\sum D\right)^{2}}{N}
$$

و. يبحث الباحث عن (SE

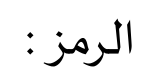

$$
S E_{M D}=\frac{S D_{D}}{\sqrt{N-1}}
$$

ز. يبحث الباحث عن to باستعمال

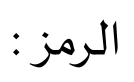

في هذا البحث استعمل الباحث

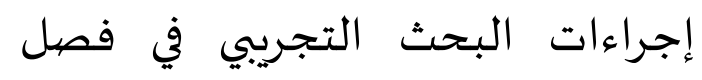
السابع (أ) هو عملية تعليم مهارة القراءة الجراء باستخدام وسيلة الأوراق الممزقة، وعملية تعليم مهارة القراءة بلدون

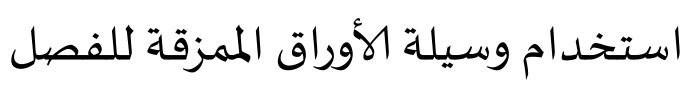
السابع (ب). ثم يقارن الباحث بين

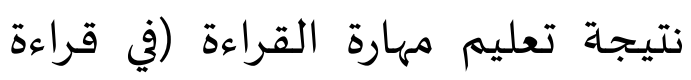
النصوص العربية وفهم المقروء) باستخدام وسيلة الأوراق الممزقة قبل أن يطبقها الباحث و بعد أن يطبقها. وبعد ذلك، وجد الباحث تأثير استخدام وسيلة الأوراق الممزقة في تعليم مهارة الباحت تاتير استخدام

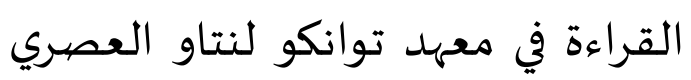
المتحدي. بعد ما قام الباحث بالاختبارين يعني اختبار قبلي واختبار بعدي في

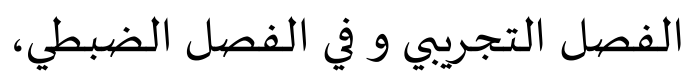
وجد الكاتب النتيجة وفصحها وحللها تحليلا إحصائيا لتعريف تأثير استخدام وسيلة الأوراق الممزقة على مهارة القراءة ثم جعلها أسـاسية في استنباط الخلاصية

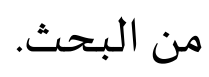
وفي إنهاء البيانات يستعمل الكاتب تحليل اختبار-ت (Test “t”) وهي لهي

$$
\stackrel{\mathrm{t}_{\mathrm{o}}=\mathrm{MD}}{\mathrm{S} \mathrm{E}_{\mathrm{MD}}}
$$

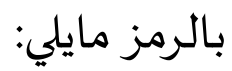

$$
\mathrm{t}_{\mathrm{o}}=\underline{\mathrm{MD}}
$$




\begin{tabular}{|c|c|c|c|c|}
\hline 171 & 19 & 07 & Vo & $r$ \\
\hline $1 \varepsilon \varepsilon$ & $1 T$ & ת & Vo & $\varepsilon$ \\
\hline$\varepsilon \ldots$ & $r$. & 00 & Vo & 0 \\
\hline VYq & rV & 01 & 10 & 7 \\
\hline$\varepsilon \ldots$ & $r$. & V. & 9. & $V$ \\
\hline V^乏 & r人 & $7 V$ & 90 & $\Lambda$ \\
\hline$\varepsilon \wedge \varepsilon$ & rr & $7 \Lambda$ & 9. & 9 \\
\hline VYq & TV & $7 \Lambda$ & 90 & 1. \\
\hline Tro & 10 & 70 & $\Lambda$. & 11 \\
\hline $1 . r \varepsilon$ & rr & 01 & 9. & 14 \\
\hline $1 . r \varepsilon$ & r & سד & 90 & $\mu$ \\
\hline$\varepsilon \wedge \varepsilon$ & rr & & 10 & $1 \varepsilon$ \\
\hline 1497 & r & $\varepsilon q$ & 10 & 10 \\
\hline $1 . r \varepsilon$ & r & س & 10 & 17 \\
\hline $1 . r \varepsilon$ & r & س & 10 & $1 \mathrm{~V}$ \\
\hline $10 Y 1$ & $r_{q}$ & $\varepsilon q$ & 9. & 11 \\
\hline r. ro & $\leqslant 0$ & $\varepsilon 0$ & 9. & 19 \\
\hline $9 \ldots$ & r. & 0 . & $\Lambda$. & $r$. \\
\hline $\begin{array}{l}\sum \mathbf{D}^{2}= \\
17719\end{array}$ & $\begin{array}{c}\sum \mathrm{D}= \\
001\end{array}$ & $\begin{array}{l}\sum \mathrm{Y}= \\
11 \leqslant V\end{array}$ & $\begin{array}{l}\sum \mathbf{X}= \\
i v . .\end{array}$ & $\begin{array}{c}\mathbf{N}= \\
\mathrm{r} .\end{array}$ \\
\hline
\end{tabular}

$$
\text { ح. إعطاء التفسير لنتيجة to }
$$

كان تحليل البيانات بين

نتيجة الفصل التجريبي و الفصل الضبطي تحليلا أخيرا من هذا البحث. كما هو المعروف أن أهداف هني هين اخديراف لتعليم مهارة القراءة في هذا البحث هو لقراءة النصوص العربية ، فحلل الكاتب بيانات بخطوات إحصائية من إختبار - ت. و حللها الكاتب من بن نتيجة الإختبار من تعليم مهارة القراءة باستخدام وسيلة الأوراق الممزقة في فصل في التجريبي ونتيجة الإختبار من تعليم مهارة القراءة بدونها في فصل الضبطى (Y).

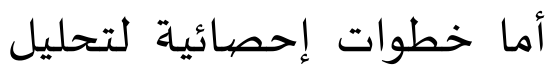
البيانات من قراءة النصوص العربية فهي باختبارات، كما يلي:

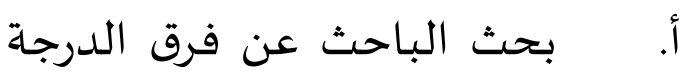
بين النتيجتين باستعمال الرمز:

$$
\mathrm{D}=\mathrm{X}-\mathrm{Y}
$$

ج. بحث الباحث عن معدل فرق الدرجة باستعمال الرمز :

ب. بحث الكاتب عن درجة D حتى يحصل على

\begin{tabular}{|c|c|c|c|c|}
\hline \multicolumn{5}{|c|}{ الجدول الثاني } \\
\hline $\mathbf{D}^{2}$ & D & $(\mathrm{Y})$ & $(\mathrm{X})$ & تلميذذ \\
\hline$\varepsilon \varepsilon 1$ & YI & 09 & $\Lambda$. & 1 \\
\hline $17 \ldots$ & $\varepsilon$. & ro & Vo & r \\
\hline
\end{tabular}

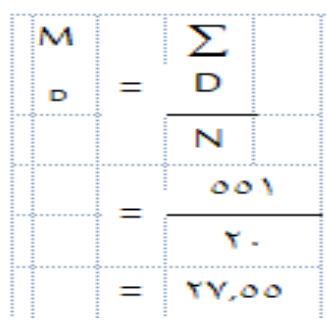


ز. بحث الباحث عن to باستعمال الرمز

$\begin{aligned} t_{0} & =\frac{M D}{S E_{M D}} \\ & =\frac{T V, 00}{1,9 \Sigma} \\ & =1 \Sigma, T\end{aligned}$

ح. إعطاء التفسير لنتيجة to

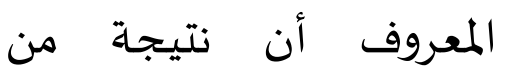

التحليل الإحصائي لاختبار-ت هي

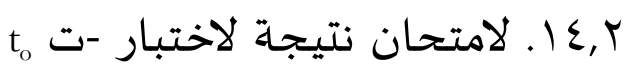
باستشارة إلى قيمة t، كما في الجدول على الدرجة ا ب \%. وإذا كان نتيجة

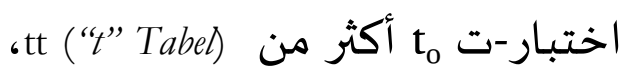

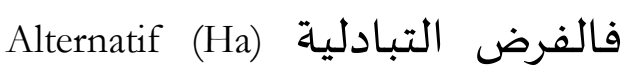
مقبولة والفرض العدمية Nihil (Ho) مردودة، وبالعكس إذا كان أقل من ولن tt (“t” Tabeh) مردودة والفرض العدمية مقبولة. أساسا على الحاصل تحليلا إحصائيا أن نتيجة to $t_{0}$

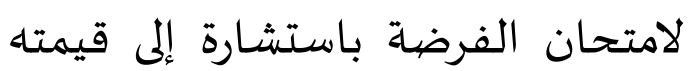

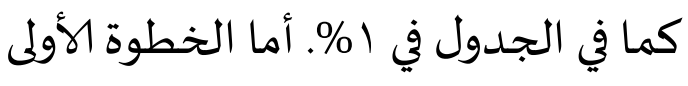

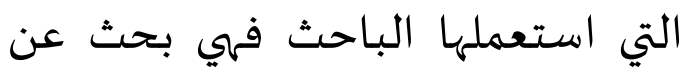
قيمة أو نتيجة درجة حرورية (db).
د. ضياعف الباحث درجة الفرق D ثم يبلغها حتى يحصل درجة DD هـ بحث الكاتب عن الانحراف المعياري Defiasi Standar dari ( عن فرق الدرجة (Difference [SD $\left.{ }_{\mathrm{D}}\right]$

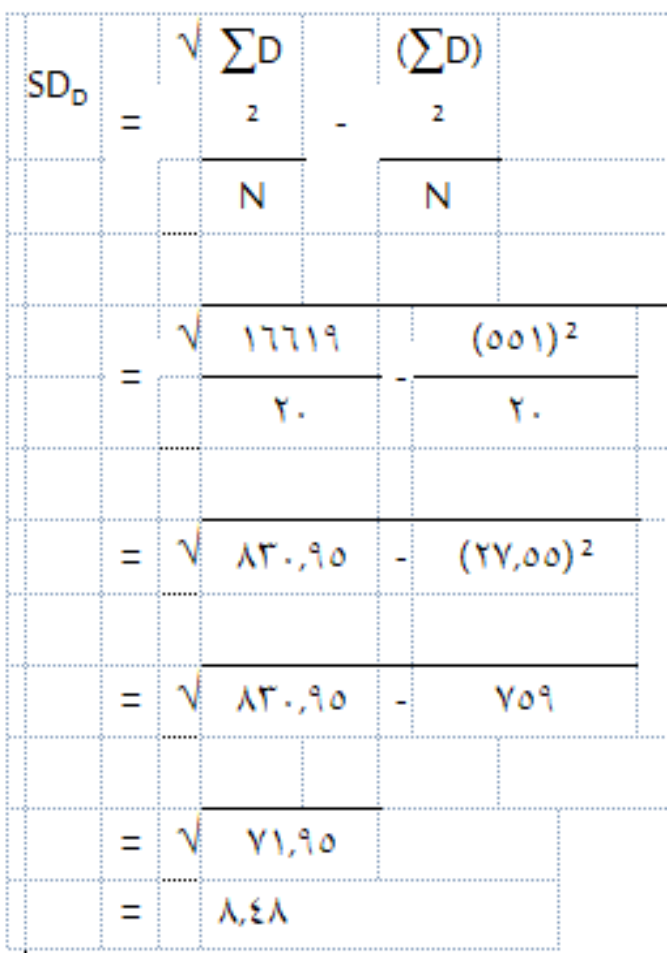

و. بحث الباحث عن (SEMD) باستعمال

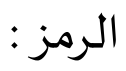


صحيحة ويعبروا أفكارهم صحيحة. واهتم الباحث نتيجتها منخفضية. فقام الباحث البحث عن وسيلة الأوراق الممزقة وتأثيرها على مهارة القراءة. بعد لبحت مااستخدم الباحث وسيلة الأوراق الممزقة في التعليم فوجد الباحث نتيجة التعليم بالنتيجة المرتفعة. ونظوه الباحث بتحليل نتيجة الاختبار. إضافة على ذلك، فظهر الباحث

مقارنة نتيجة التلاميذ بين الفصل

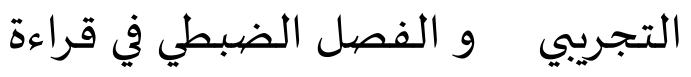
النصوص العربية، وهي كما في الجدول

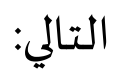

\begin{tabular}{|c|c|c|c|}
\hline \multicolumn{4}{|c|}{ الجـدول الثالث } \\
\hline \multicolumn{4}{|c|}{ Statistics } \\
\hline & & $x$ & $\mathrm{Y}$ \\
\hline \multirow[t]{2}{*}{$N$} & Valid & 20 & 20 \\
\hline & Missing & 0 & 0 \\
\hline \multicolumn{2}{|c|}{ Mean } & 85 & 57.35 \\
\hline \multicolumn{2}{|c|}{ Median } & 87.50 & 58.00 \\
\hline \multicolumn{2}{|c|}{ Mode } & 85 dan 90 & 63 \\
\hline \multicolumn{2}{|c|}{ Minimum } & 75 & 35 \\
\hline \multicolumn{2}{|c|}{ Maximum } & 95 & 70 \\
\hline \multicolumn{2}{|c|}{ Sum } & 1700 & 1147 \\
\hline
\end{tabular}

تصور الجدول السابق أن عدد

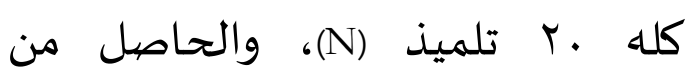
مجموعاة نتيجة في قراءة النصوص

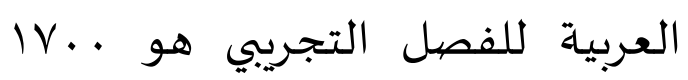
(XX)، والحاصل من مجموعة في قراءة
إذا كان N عينة في هذا البحث هي بان

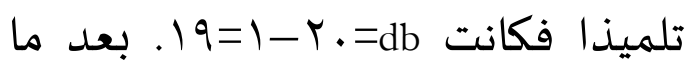
اهتم الكاتب جدول نتائج،، فالمعروف أن

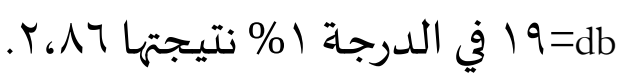
كانت نتيجة to قدرة التلاميذ على قراءة النصوص العربية في الفصل

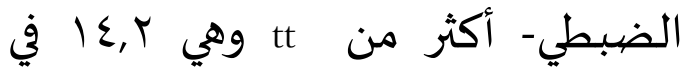
الدرجة |٪ فالفروض العدمية مردودة الهن والفروض التبادلية مقبولة. إذن، وجد الكاتب فرقا بين قدرة التلاميذ فى قراءة النصوص العربية من مهارة القراءة

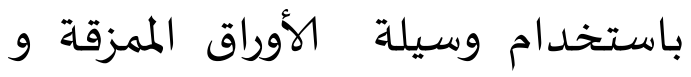

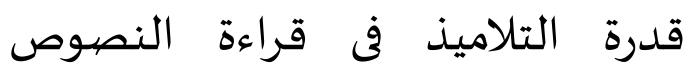
العربية بلدون استخد امها،

إن تعليم اللغة العربية أربع

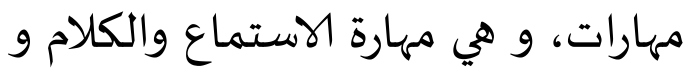

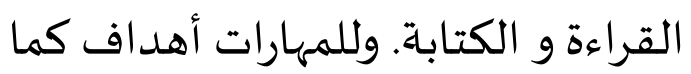
في 2008 Permenag No. 2 Tahun . كان في معهد توانكو لنتاو العصري المتحدي

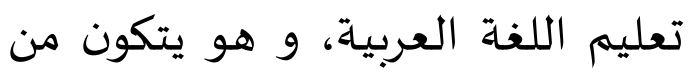

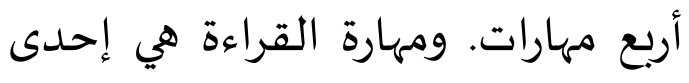
المهارات التي تعلمها التلاميذ فيها.

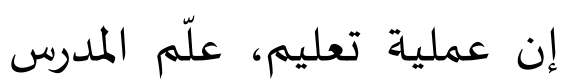
مهارة القراءة في هذا المعهد بدون

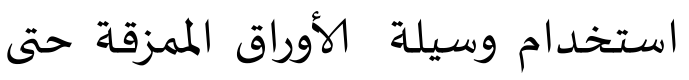

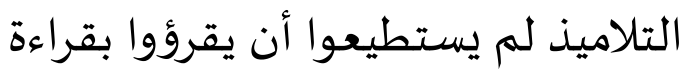


التجريبي هو • • ال (XX)، والحاصل من مجموعة في فهم المقروء للفصل هون مهن

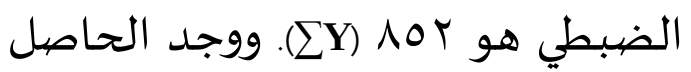
بمعدل (Mean) للفصل التجريبي

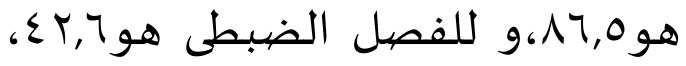
وأكبر القيمة (Max) للفصل التجريبي هو

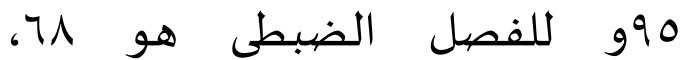
وأقلالقيمة (Min) للفصل التجريبيهو هVو للفصل الضبطي هو .. والوسيط للفصل التجريبي هو (Median) للفصل الضبطي هو 1.0؛، والموضة للفصل التجريبي هو 10، و و و للفصل الضبطي هو rr. بتحليل البحث ظهر أن هناك اختلاف النتيجة بين التلاميذ الذين علمهم المدرس باستخدام وسيلة الأوراق الممزقة والتلاميذ الذين علمهم بهم المدرس بدون استخدام وسيلة الأوراق الممزقة في مهارةالقراءة. وكذلك أن بلن

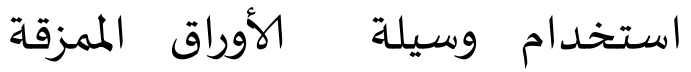
يستطيع أن يؤثر على مهارة القراءة في معهد توانكو لنتاو العصرى المتحدي.

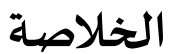

استخدام وسيلة الأوراق الممزقة يؤثر على قدرة التلاميذ في قراءة النصوص العربية من مهارة القراءة،
النصوص العربية للفصل الضبطي هو ل الحاصل بمعدل (Mean) للفصل التجريبي هومي، و للفصل الضبطي هو Or,OV، وأكبر القيمة (Max) للفصل التجريبي هو 90 و للفصل الضبطي هو . V، وأقل القيمة (Min) للفصل التجريبي هو V0 وللفصل الضبطي هو هr. والوسيط للفصل التجريبي هو .NV,0 (Median) للفصل الضبطي هو ..,01 والموضة

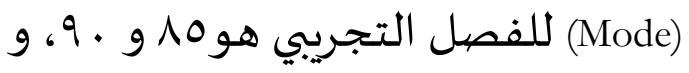
للفصل الضبطي هوسا7. وكذلك في فهم المقروء، فظهر هوت الباحث مقارنة نتيجة التلاميذ بين

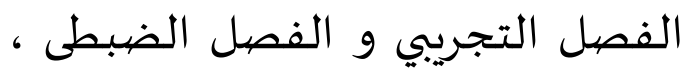
وهي كما في الجدول التالي:

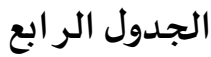

Statistics

\begin{tabular}{|c|c|c|c|}
\hline & & $x$ & $\mathrm{Y}$ \\
\hline \multirow[t]{2}{*}{$N$} & Valid & 20 & 20 \\
\hline & Missing & 0 & 0 \\
\hline \multicolumn{2}{|c|}{ Mean } & 86.5 & 42.60 \\
\hline \multicolumn{2}{|c|}{ Median } & 48.5 & 48.5 \\
\hline \multicolumn{2}{|c|}{ Mode } & 85 & 32 \\
\hline \multicolumn{2}{|c|}{ Minimum } & 75 & 0 \\
\hline \multicolumn{2}{|c|}{ Maximum } & 95 & 68 \\
\hline \multicolumn{2}{|c|}{ Sum } & 1730 & 852 \\
\hline
\end{tabular}

تصيور الجدول السابق أن عدد

كله ب تلميذ (N)، والحاصل من مجموعة نتيجة في فهم المقروء للفصل 
سالم، محمد و سرايا، عادل السيد أحمد.

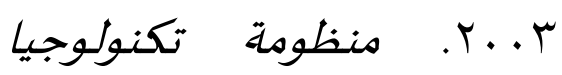

التعليم .الرياض: مكتبة الرشد.

سلامة، عبد الحافظ محمد .عY乏 هـ تصيميم التدرلي. الرياض: دار الخريدين.

السمان، محمود على. به191. التوجيا في تلدريس اللغة العـبية. القاهرة:

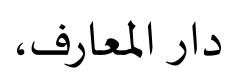

صينى، محمد إسماعيل و عبد الله، عمر

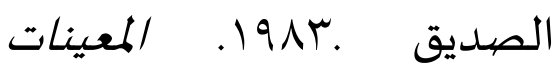

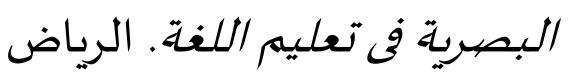
جامعة الملك سعود.

طعيمة، رشدى أحمد. 1910. دليل عمل في إعداد المواد التعلمية احملية لبراهج تعليم العربية. مكاة

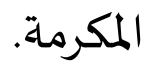

العزاوي، رحيم يونس كرو .V.... مقلدمة في منهج البحث العلمي. عمان: دار دجلة.

مدكور، علي أحمد .9. . . . . تلدريس فنون اللغة العربية النظرية
وهذا يستدل على النتيجة التى حصلها الباحث بين الفصل التجريبي والفصل

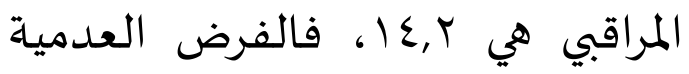
مردودة والفرض التبادلية مقبولة.

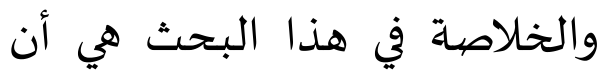
وسيلة الأوراق الممزقة تستطيح أن تؤثر

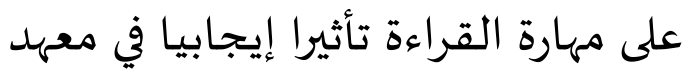
توانكو لنتاو العصري المتحدي، وهذا تدل على أن التعليم باستخدام وسيلة الأوراق الممزقة يرقي نتيجة التلاميذ في

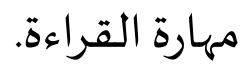

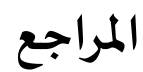
إبرهيم، عبد العليم .119. الموجع الفنى لمدريسى اللغة العربية. القاهرة :دار المعارف.

بدري، أحمد زكي. 1911. معجم الإصطلاح العلوم الاجتماعية. يروت: مكتبة لبنان.

الحيلة، محمد محمود .0. . ب. تكنولوجيا

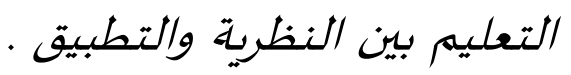
عمان: دار المسيرة للنشر لنشئ والتوزيع. 
يونس، محمود و بكرى، قاسم .190 1.

التوبية والتعليهم · بوكت تنبجي:

$$
\text { نوسنتار. }
$$

Ainin, M. 2007. Metodologi Penelitian Babasa Arab. Malang: Hilal Pustaka.

Arsyad, A. 2005. Media Pembelajaran. Jakarta: PT Raja Grafindo Persada.

Hermawan, A. 2011. Metodologi Pembelaran Bahasa Arab. Bandung: Remaja Rosda Karya.

Machmudah, U. \& Rosyidi, A. W. 2008. Active Learning dalam Pembelajaran Bahasa Arab. Malang: UIN Malang Press.

Sudijono, A. 2005. Pengantar Statistik Pendidikan. Jakarta: Raja Grafindo Persada.

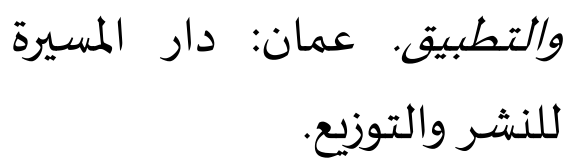

